\title{
Mot du rédacteur en chef
}

C'est avec grand plaisir que je vous présente ce nouveau volume des Cahiers de l'ILOB, qui contient les actes choisis du colloque du CCERBAL de 2016, intitulé «Bilingue dès la naissance : processus, pédagogie et politique ». Je tiens à remercier les rédacteurs invités, Christopher Fennell et Richard Clément, pour la supervision des processus de sélection et de relecture d'articles. J'aimerais également saluer le travail de Bojan Lalovic, documentaliste du Centre de ressources Julien-Couture, celui des assistantes éditoriales et traductrices, Myriam Ducos, Andréanne Allard-Blanchard, et Sydney Goudreau-Patterson, ainsi que celui de la rédactrice responsable de la production et de la vérification de références, Christina Thiele. Bonne lecture !

Nikolay Slavkov

Directeur

Centre canadien d'études et de recherche en bilinguisme et aménagement linguistique (CCERBAL)

Ottawa, juillet 2017 